\title{
OPTIMIZACIÓN DE LA VIDA DE BATERÍAS EN PLANTAS FOTOVOLTAICAS MEDIANTE ALGORITMOS GENÉTICOS
}

\author{
E. Pérez, H. Beltrán, C. Ariño, J.C. Alfonso \\ Dep. de Ingeniería de Sistemas Industriales y Diseño, Universidad Jaume I, Castellón de la Plana, España \\ \{pereze, hbeltran, arino, jalfonso\}@uji.es
}

\begin{abstract}
Resumen
Este artículo analiza el problema de la reducción del envejecimiento producido en baterías cuando éstas se utilizan en un una planta fotovoltaica $(F V)$ que participa en el mercado de electricidad $y$, por tanto, debe generar de una manera controlada en 24 escalones horarios para satisfacer la producción comprometida en las pujas de mercado. El trabajo presentado propone una estrategia basada en una optimización mediante algoritmos genéticos para la determinación de esos 24 escalones de potencia de manera que el envejecimiento que se produce, calculado mediante un algoritmo de Rainflow counting, sea mínimo, al mismo tiempo que se vierte a la red toda la potencia generada por la planta. Se ofrecen resultados de simulación, a partir de datos de generación FV obtenidos de una base de datos europea, que muestran que la estrategia propuesta produce un envejecimiento en la batería menor que el que se obtiene con otras técnicas de optimización.
\end{abstract}

Palabras clave: Baterías, Rainflow counting, envejecimiento, integración fotovoltaica, algoritmos genéticos.

\section{INTRODUCCIÓN}

Últimamente, los operadores de sistema eléctrico se enfrentan a nuevos e interesantes desafíos para equilibrar las redes eléctricas. Esto es debido principalmente a la introducción de un gran número de generadores basados en tecnología renovable, con una producción altamente estocástica [1], además de la tradicional variabilidad de la demanda de energía. Ambas circunstancias comprometen las metodologías actualmente utilizadas para el control de dichas redes, haciendo cada vez más difícil conseguir el equilibrio del sistema a la vez que se mantienen sus parámetros de operación (tensión, frecuencia, etc..) en rangos apropiados.

En este sentido, la variabilidad de la generación de ciertas fuentes renovables, como los aerogeneradores o las placas fotovoltaicas, supone una dificultad para su integración en el sistema eléctrico. Aun- que actualmente dichas fuentes representan una pequeña fracción en los mixes energéticos de la mayoría de países, el incremento de la capacidad instalada [2] obligará a las instalaciones futuras no sólo a producir tanta energía activa como sea posible, sino también a participar en el mercado eléctrico y, potencialmente, dar soporte a la red ofreciendo servicios complementarios. Esto implica la necesidad de una producción más fiable y predecible y abre la puerta al uso de tecnologías de generación híbrida, que integren sistemas de almacenamiento de energía en plantas fotovoltaicas [3].

Existen ya varios estudios en esta dirección, con el objetivo de alcanzar una producción más constante en sistemas fotovoltaicos. Entre otros, se propone combinar estos sistemas con diferentes tecnologías como: pilas de hidrógeno [4], aerogeneradores [5] o, en mayor medida, baterías $[6,7,8,9,10]$. Sin embargo, estos últimos estudios se han enfocado generalmente a redes de pequeño tamaño o sistemas fotovoltaicos aislados, o para modificar o suavizar la producción fotovoltaica de algún modo.

En este artículo se analiza la integración de baterías en una planta fotovoltaica de gran tamaño conectada a red que opera en el mercado eléctrico. Para ello, se debe asegurar una producción energética que se adecue a la oferta realizada por el operador de la planta en la sesión de mercado anterior puesto que, si existen desviaciones, éstas suponen penalizaciones económicas.

Un aspecto clave que puede llegar a comprometer la viabilidad económica de una planta fotovoltaica con almacenamiento es la amortización del coste de las baterías a utilizar, que depende directamente de la vida de éstas. Los dos factores más críticos para ello son el número de ciclos de carga y descarga y la profundidad de estos, es decir, la operación que se realice en funcionamiento de la batería.

En este artículo se pretende, para una evolución de la potencia fotovoltaica conocida, determinar la manera óptima en la que debe comprometerse el suministro a la red de dicha potencia desde el punto de vista del desgaste de las baterías. 
El artículo está organizado de la siguiente forma: en primer lugar, en la sección 2 se describen los tipos de configuración de mercados de electricidad existentes y la utilización de baterías en plantas fotovoltaicas para adaptarse a ellos. A continuación, la sección 3 describe la técnica utilizada para el recuento de ciclos de carga y descarga experimentados por una batería sometida a patrones irregulares, así como la evaluación del envejecimiento que éstos producen. En la sección 4 se plantea el problema de la determinación de una secuencia de potencias para participación en mercado, introduciendo las alternativas básicas para ello. Por último, se plantea una nueva estrategia para la determinación de esta secuencia basada en la optimización mediante algoritmos genéticos y se ofrecen unas conclusiones.

\section{INTEGRACIÓN DE PLANTAS FV EN EL MERCADO}

\subsection{CONFIGURACIÓN DE LOS MERCADOS DE ELECTRICIDAD}

Como consecuencia del proceso de reestructuración experimentado por los mercados de electricidad en todo el mundo durante los últimos 20 años, se han adoptado diferentes configuraciones para alcanzar un entorno competitivo. Sin embargo, en la mayoría de países se ha establecido una estructura que combina mercados a futuro y en tiempo real. Desde este punto de vista, y de acuerdo al modo de negociación, éstos se pueden dividir entre los que presentan un modelo de negociado en una cámara de compensación ( $p o o l$ ), los que proponen un modelo con contrato bilateral y los que tienen un modelo híbrido [11]. Por lo tanto, mientras que en el mercado pool se negocia centralmente un intercambio de energía para todos los compradores y vendedores, en su mayoría con un día de antelación, en los modelos de contrato bilateral la electricidad se negocia directamente entre los participantes del mercado de manera individual.

Un desafío clave para ambos tipos de estructura de mercado viene dado por las interrupciones inesperadas en la generación o los cambios no previstos en la demanda. Estos fenómenos pueden producirse en cualquier momento entre el cierre del mercado de electricidad, o el momento de establecimiento del contrato, y la entrega de energía programada en el día siguiente. Para corregir estas contingencias repentinas, la mayoría de los sistemas cuentan con un mercado paralelo "intradiario" que permite a los participantes del mercado negociar más cerca del momento de entrega, hasta justo antes del cierre. En este sentido, los mercados intradiarios son en muchas ocasiones un mer- cado continuo que se abre varias veces al día, desde 6 hasta 24 dependiendo del país, con un cierre entre una y tres horas antes del período de entrega de la energía [12].

En el presente trabajo se considera el Mercado Ibérico de la Electricidad (MIBEL) que presenta una estructura de tipo pool con periodos operacionales de entrega de energía de una hora y seis sesiones de mercado intradiario, Fig. 1. Por tanto la producción de la planta fotovoltaica con almacenamiento debe optimizarse para poder negociar con garantías en este escenario.

\subsection{USO DE BATERÍAS PARA LA OPERACIÓN DE PLANTAS FV EN EL MERCADO}

Como se ha visto, si la tecnología FV continúa su tendencia actual, no tardará en suponer una parte importante del mix energético en ciertos sistemas eléctricos de todo el mundo. Esto implica la necesidad de que esta tecnología participe en el mercado eléctrico, tal y como hacen las fuentes convencionales, y obligará por tanto a las plantas FV a reducir su intermitencia inherente, no sólo para cumplir los requisitos que establece el operador de la red, sino también para minimizar los desvíos de la producción respecto a la energía negociada en el mercado, reduciendo así las penalizaciones económicas. De acuerdo a las configuraciones de mercado descritas, las plantas FV deberán ser capaces de entregar su producción de manera constante y controlada, normalmente durante períodos horarios. Para ello, será necesaria la inclusión de algún tipo de tecnología de almacenamiento en la planta FV.

Entre las posibles soluciones de almacenamiento actualmente disponibles, no todas se adaptan de manera adecuada a las instalaciones FV [13]. Algunas, como los bombeos hidráulicos o los sistemas de almacenamiento en aire comprimido, presentan inconvenientes tales como la dependencia de una localización adecuada. Otras, como los supercondensadores o los volantes de inercia, tienen una capacidad de almacenamiento limitada. Opciones como el almacenamiento energético super magnético aún no son suficientemente maduras. Por último, opciones como el almacenamiento térmico están fuera del alcance de las plantas FV. Por tanto, puede concluirse que las diferentes familias de baterías que se están desarrollando y comercializando hoy en día son el tipo de sistema apropiado para su utilización en una aplicación de este tipo.

Aunque actualmente varios tipos de baterías han alcanzado una madurez en su desarrollo suficiente como par ser consideradas soluciones viables técni- 


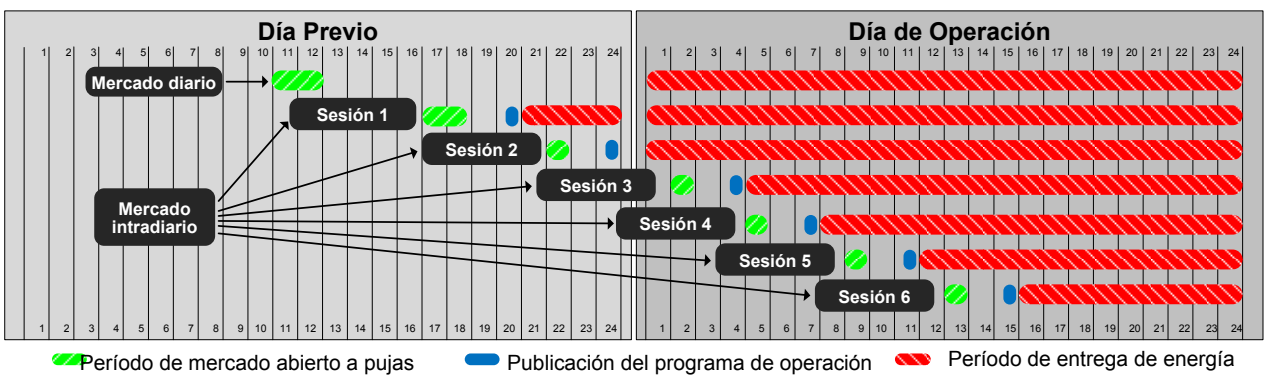

Figura 1: Programación de los mercados diario e intradiarios en MIBEL.

camente para su uso en plantas FV, sin duda entre ellas destaca la tecnología de iones de litio. Esto se debe principalmente a que ya han sido usadas con éxito en la industria del vehículo eléctrico (con compañías como Tesla Motors a la cabeza). De hecho, esta tecnología ha sido la implantada en la mayoría de las instalaciones residenciales existentes. Por todo ello, esta será la tecnología considerada en las simulaciones propuestas en el presente trabajo.

\section{CÁLCULO DEL ENVEJECIMIENTO DE BATERÍAS}

El proceso de degradación de los diferentes tipos de batería ha sido estudiado en muchos trabajos de investigación. La mayoría de ellos identifican como los factores más influyentes el número de ciclos de carga-descarga experimentados, la temperatura de operación, las velocidades de carga y descarga, la profundidad de descarga de los ciclos, el estado de carga típico de la batería, la tensión límite de carga y la energía total procesada $[9,11,14]$.

No obstante, no resulta evidente el desarrollo de un modelo general que incorpore todos estos factores para el análisis del proceso de desgaste de las baterías en el contexto de aplicaciones fotovoltaicas. Los distintos factores tendrán diferente efecto dependiendo del tipo de batería. Además, algunos de ellos variarán con la localización de la planta. Por tanto, teniendo en cuenta que la temperatura puede y suele controlarse de manera externa, este trabajo se centra en analizar la que comúnmente se considera la combinación más crítica de factores de envejecimiento: el numero de ciclos experimentados durante el funcionamiento y su profundidad de descarga $[15,16]$.

\subsection{RECUENTO DE CICLOS DE BATERÍA}

Una batería funcionando en paralelo con una planta FV para verter a la red una potencia constante a tramos de una hora, como se ha descrito en la sección anterior, experimentará una evolución de su estado de carga que típicamente no seguirá un patrón de ciclado regular. Por esta razón, en este trabajo se ha utilizado un algoritmo de recuento de ciclos para identificar el número de ciclos equivalente que asume la batería para cada profundidad de descarga en estas condiciones de operación. El algoritmo es el método del Rain Flow Counting $(R F C)$, que es una técnica de recuento de ciclos que se utiliza tradicionalmente en la ingeniería mecánica para el análisis de fatiga de estructuras sometidas a esfuerzos cíclicos[14]. No obstante, el RFC se ha venido aplicando recientemente también para la estimación de la vida de las baterías [17, 18], por lo que es la técnica empleada en el presente trabajo.

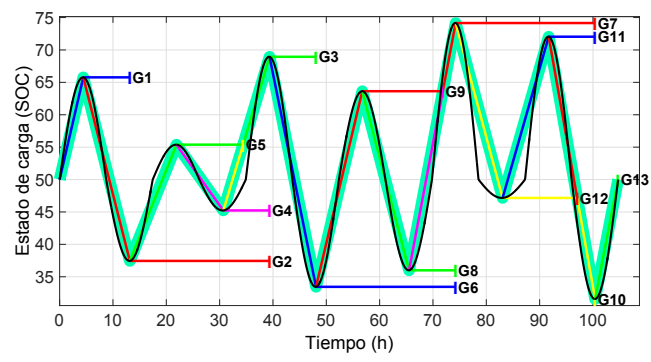

Figura 2: Curvas de ciclado resultantes (gotas en colores)

La figura 2 ilustra el funcionamiento del RFC. La linea negra es la evolución del estado de carga originalmente experimentada por las baterías y la linea azul gruesa corresponde a la adaptación que realiza el algoritmo RFC. Las lineas de color son los semiciclos de carga y descarga generados por el algoritmo, usado para contar el número de ciclos completos para cada amplitud de descarga específica. Se observa que cada semiciclo comienza en un pico máximo o mínimo. Si se gira la figura $90^{\circ}$ en sentido horario, la figura se asemeja a un tejado de pagoda con gotas de lluvia cayendo. Si se deja caer la gota desde cada máximo o mínimo, el semiciclo correspondiente a una gota específica se puede obtener siguiendo su camino por el te- 
jado hacia abajo. El flujo de cada gota ( $\mathrm{G}$ en la figura) se detiene, acabando con el semiciclo, en cualquiera de las siguientes condiciones:

1. Se encuentra con un mínimo o máximo de dirección opuesta y magnitud mayor a la del máximo o mínimo de partida. Es el caso de las gotas numeradas $1,2,3,4,6$ y 8 en la figura 2.

2. Se une al flujo de una gota que comenzó en un máximo o mínimo anterior. Es el caso de las gotas 5, 9 y 12.

3. Alcanza el instante final de la simulación. Es el caso de las gotas 7, 10, 11 y 13 .

A partir de las líneas de color de la figura 2, el algoritmo cuenta el número de gotas (es decir, semiciclos) realizados por la batería para cada profundidad de descarga, siendo la amplitud de esos semiciclos la diferencia en estado de carga entre sus puntos inicial y final. Por ejemplo, el semiciclo correspondiente a la gota 1 presenta una amplitud del $15 \%$, el de la gota 2 del $30 \%$ y el de la gota 5 del $10 \%$. Para obtener el número de ciclos completos, deben considerarse dos semiciclos de la misma amplitud y dirección diferente.

La implementación del algoritmo del RFC que se ha utilizado en el presente trabajo es una toolbox de MATLAB, descrita en [19].

\subsection{ANÁLISIS DEL ENVEJECIMIENTO}

Una vez se tiene el número de ciclos a partir del algoritmo RFC, debe compararse con la curva de capacidad de ciclado de la batería, Fig. 3. Esta curva, proporcionada por el fabricante, representa el número de ciclos que la batería puede soportar para cada posible profundidad de descarga. Dada una secuencia de evolución del estado de carga de la batería, el envejecimiento producido en la batería puede calcularse como el porcentaje de vida consumido debido a los ciclos experimentados aplicando la regla de Miner:

$$
\text { Envej. }=\sum_{D o D=1}^{D o D=100} \frac{N_{c i c}(D o D)}{N_{\max }(D o D)} \cdot 100
$$

donde $N_{\text {cic }}(D o D)$ es el número de ciclos calculados por el algoritmo RFC experimentados para cada amplitud y $N_{\max }(D o D)$ el número máximo de ciclos que la batería puede soportar a esa amplitud, de acuerdo a la curva de capacidad mostrada en la Fig. 3. En este caso, se trata del modelo "Intensium Flex Medium Power"de la batería de la marca SAFT.

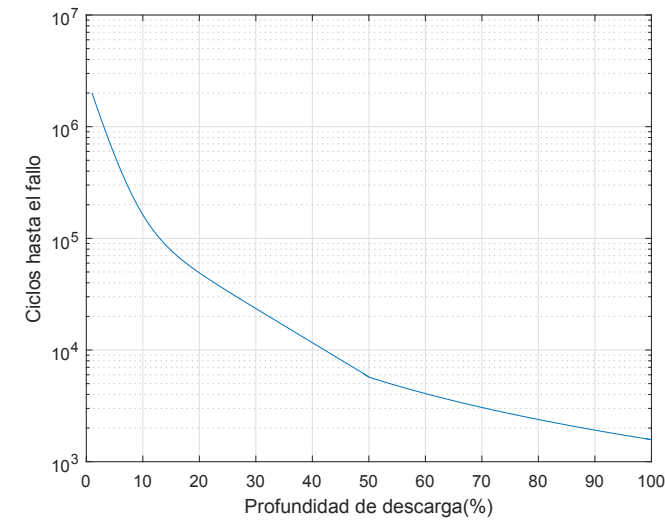

Figura 3: Capacidad de ciclado para una batería de iones de litio de la marca SAFT

Las baterías se consideran inutilizadas en la práctica cuando el envejecimento calculado de acuerdo a (1) llega al $100 \%$.

\section{PLANTEAMIENTO DEL PROBLEMA Y ESTRATEGIAS INICIALES}

A la vista de la estructura del mercado y el fenómeno del envejecimiento de las baterías resulta interesante plantearse, dada una evolución de la potencia FV conocida, qué secuencia de 24 escalones de potencia deben negociarse con el operador de la red para entregar toda la producción del panel de manera que la batería sufra un desgaste lo más bajo posible. Nótese que, en general, esta secuencia de potencias debe determinarse antes de que se cierre el mercado eléctrico, lo cuál supone que la potencia fotovoltaica producida no se conoce con exactitud. No obstante, siempre que se prevea un día claro (lo cuál es habitual en muchas de las plantas FV situadas en España), la curva de producción se puede conocer con esa antelación con mucha precisión, a partir de la localización y la fecha. En caso contrario, existen modelos de predicción a partir de diferentes variables climáticas.

Por tanto, para el presente trabajo, y como aproximación inicial, es razonable suponer que en el momento de determinar los escalones de potencia se conoce la producción fotovoltaica. Se describen a continuación dos estrategias básicas para la determinación de dichos escalones.

\subsection{POTENCIA MEDIA HORARIA}

La primera de estas estrategias consiste en establecer como potencia a entregar para cada uno de los períodos horarios negociados en el mercado la media de la potencia que produce el panel en ese 
período, tal como muestra la ecuación (2):

$P_{\text {red }}(t)=\left\{\begin{array}{l}p_{1}=\bar{P}_{F V}(1-30) t=1 \ldots 30 \\ p_{2}=\bar{P}_{F V}(31-60) t=31 \ldots 60 \\ \vdots \\ p_{24}=\bar{P}_{F V}(691-720) t=691 \ldots 720\end{array}\right.$

donde se ha considerado que se tienen datos de potencia FV cada 2 min. (720 muestras diarias), $P_{\text {red }}(t)$ es la potencia entregada a la red en cada muestra y y $\bar{P}_{F V}\left(t_{1}-t_{2}\right)$ es la media de la potencia producida por los paneles en cada período horario. La potencia a extraer de la batería puede calcularse como:

$$
P_{E S}(t)=P_{r e d}(t)-P_{F V}(t)
$$

Intuitivamente, esta parece una estrategia lógica para no cargar ni descargar más de lo necesario la batería.

\subsection{MINIMIZACIÓN DE LA DESVIACIÓN DEL ESTADO DE CARGA}

Esta estrategia, explicada en detalle en [20], consiste en una optimización cuadrática (QP) cuyo objetivo es mantener en todo momento el estado de carga de la batería lo más cerca posible de un estado de carga de referencia (generalmente el $50 \%$ ), de forma que se requiera una capacidad de almacenamiento pequeña. Como se explica en el trabajo citado, la optimización a resolver es

$$
\min \sum_{0}^{n}\left(S O C(t)-S O C_{r e f}\right)^{2}
$$

sujeto a (para $t=1 \ldots 720)$

$$
\begin{gathered}
P_{r e d}(t)=\left\{\begin{array}{l}
p_{1} t=1 \ldots 30 \\
p_{2} t=31 \ldots 60 \\
\vdots \\
p_{24}=t=691 \ldots 720
\end{array}\right. \\
P_{r e d}(t)=P_{F V}(t)+P_{E S}(t) \\
P_{E S, \text { min }} \leq P_{E S}(t) \leq P_{E S, \max } \\
E_{E S}(t)=E_{E S}(t-1)-T P_{E S}(t) \\
E_{E S, \min } \leq E_{E S}(t) \leq E_{E S, \max }
\end{gathered}
$$

donde $S O C(t)$ es el nivel de carga de la batería en cada instante de muestreo expresado porcentualmente respecto a la capacidad de la batería, y $S O C_{r e f}$ el valor de referencia.

Este problema QP calcula los 24 escalones de potencia, usados como referencia para la planta, sujeto a cuatro restricciones: las restricciones (4) y

\begin{tabular}{|c|c|c|}
\hline & Marzo & Abril \\
\hline P. media & 0.0491 & 0.0446 \\
\hline Min. SOC & 0.0614 & 0.0382 \\
\hline
\end{tabular}

Tabla 1: Desgaste de baterías en un día (\%)

(5) establecen, respectivamente, el funcionamiento de la planta y limitaciones en las referencias de potencia de la batería. En cuanto a (6) y (7), definen la evolución del estado de carga a lo largo del día y limitan su valor entre un mínimo y un máximo.

Además, para el presente trabajo se añade una restricción adicional:

$$
S O C(720)=S O C(1)
$$

Esta restricción deja el sistema de almacenamiento con el mismo nivel de carga inicial, para que se entregue a la red toda la potencia entrante en los paneles.

\subsection{CASOS DE ESTUDIO}

Puesto que las dos estrategias propuestas parecen adecuadas con el objetivo de producir un desgaste bajo en la batería, a continuación se comparará, aplicando el algoritmo de RFC descrito en la sección 3 , el desgaste que se produce con cada una de ellas en una batería de $0.1 \mathrm{MWh}$ funcionando en una planta de $1 \mathrm{MW}$, para algunos días en los que se tiene una producción fotovoltaica diferente.

Para ello, se tomarán datos para una localización concreta de la base de datos de la Unión Europea PVGIS (Photovoltaic Geographical Information System) [21]. Dicha base de datos se utiliza habitualmente para analizar la viabilidad económica a largo plazo de plantas FV puesto que proporciona datos de producción FV estadísticos. En PVGIS pueden encontrarse promedios mensuales de curvas de producción diaria, muestreadas cada 15 minutos.

Se escoge en primer lugar el promedio del mes de marzo y se aplican las dos estrategias, obteniéndose las potencias óptimas y las evoluciones del estado de carga que pueden observarse en la figura 4. Análogamente, se aplican las dos estrategias para el promedio del mes de abril obteniéndose una curva de potencias muy similar y la evolución del estado de carga mostrada en la Fig. 5.

Como se ha visto, a partir de esta evolución del estado de carga puede utilizarse el algoritmo del RFC y la ecuación (1) para calcular el desgaste producido en la batería. De este modo, se obtienen los resultados mostrados en la tabla 1.

Puesto que, para los dos casos analizados, los re- 

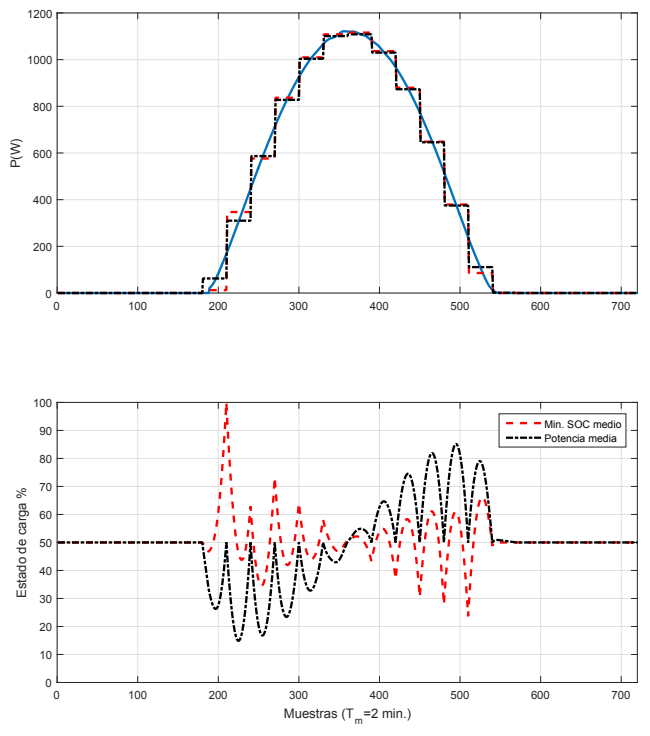

Figura 4: Potencias y estados de carga (marzo)

sultados obtenidos no permiten elegir una estrategia sobre la otra para la minimización del desgaste de la batería, se amplía el estudio para los 12 días tipos de los 12 meses de los que se dispone de datos en PVGIS. Estos resultados (que están graficados junto a los resultados finales en la figura 7) confirman que ninguna de las dos estrategias es claramente superior a la otra en términos del desgaste de la batería, pues los resultados difieren dependiendo del día en concreto. Por ello, en la siguiente sección se va a plantear una nueva estrategia que calcule una nueva secuencia de potencias teniendo directamente como objetivo la minimización del envejecimiento. A la vista de la forma que se tiene de determinar este envejecimiento, con una dependencia fuertemente no lineal en la evolución del estado de carga, se opta por enfocar el problema con ayuda de algoritmos genéticos.

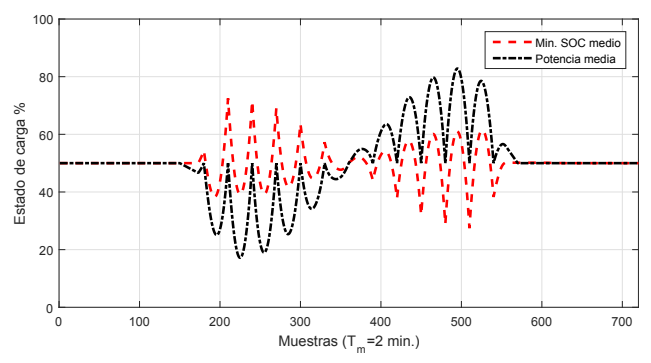

Figura 5: Estados de carga (abril)

\section{OPTIMIZACIÓN MEDIANTE ALGORITMOS GENÉTICOS}

Un algoritmo genético (AG) es un método inspirado en la evolución biológica que permite resolver problemas de optimización con restricciones [22]. El AG modifica repetidamente una población de soluciones individuales. En cada paso, el AG selecciona aleatoriamente individuos de la población actual para ser padres y los usa para producir hijos para la siguiente generación, de forma que la población "evolucione" hacia una solución óptima. Los AG pueden aplicarse a una gran variedad de problemas de optimización, incluyendo aquellos que presentan una función objetivo discontinua o altamente no lineal. Este es, precisamente, el caso de una función de coste que represente el envejecimiento de una batería calculado mediante RFC.

La optimización que se pretende resolver es:

$$
\begin{gathered}
\text { mín Envej. }=R F C\left(p_{1}, \ldots, p_{24}\right) \\
\text { sujeto a }(\text { para } t=1 \ldots 720) \\
P_{\text {red }}(t)=\left\{\begin{array}{l}
p_{1} t=1 \ldots 30 \\
p_{2} t=31 \ldots 60 \\
\vdots \\
p_{24}=t=691 \ldots 720
\end{array}\right. \\
P_{\text {red }}(t)=P_{F V}(t)+P_{E S}(t) \\
P_{\text {min }} \leq P_{r e d}(t) \leq P_{\text {max }} \\
E_{E S}(t)=E_{E S}(t-1)-T P_{E S}(t) \\
E_{E S, \text { min }} \leq E_{E S}(t) \leq E_{E S, \text { max }}
\end{gathered}
$$

donde $\operatorname{RFC}\left(p_{1}, \ldots, p_{24}\right)$ representa la función no lineal que realiza el cálculo del envejecimiento de la batería aplicando el método del Rainflow Counting y la ecuación (1) tal como se describe en la sección 3.

El AG se implementa mediante la Optimization toolbox de MATLAB y se configura con las siguientes opciones:

- Población de 200 individuos de vectores reales.

- Selección estocástica uniforme de individuos que contribuyen a la siguiente generación.

- Élite del 5\% de individuos que sobreviven hasta la siguiente generación.

- $80 \%$ de individuos nuevos producidos por cruce y el resto por mutación.

- El cruce crea los nuevos individuos mediante una media con ponderación aleatoria y la mutación generando direcciones aleatorias adaptativas en función del éxito de la generación anterior. 
Además, se utiliza una población inicial que incluye las soluciones obtenidas con las dos estrategias básicas descritas en la sección anterior. Por la propia filosofía de funcionamiento del AG, esto nos garantiza que siempre conseguirá una solución que al menos iguale la mejor de las dos opciones y, en la gran mayoría de los casos, la mejorará.

La figura 6 muestra las mismas curvas mostradas en la figura 4 pero añadiendo la nueva solución obtenida mediante el AG. Puede comprobarse que la profundidad de los ciclos de carga y descarga es algo más suave que con la estrategia de potencia media. Si se calcula el desgaste que se produce con la solución obtenida mediante el AG, este es del $0.0454 \%$, frente al $0.0491 \%$ y $0.0614 \%$ de las estrategias básicas.
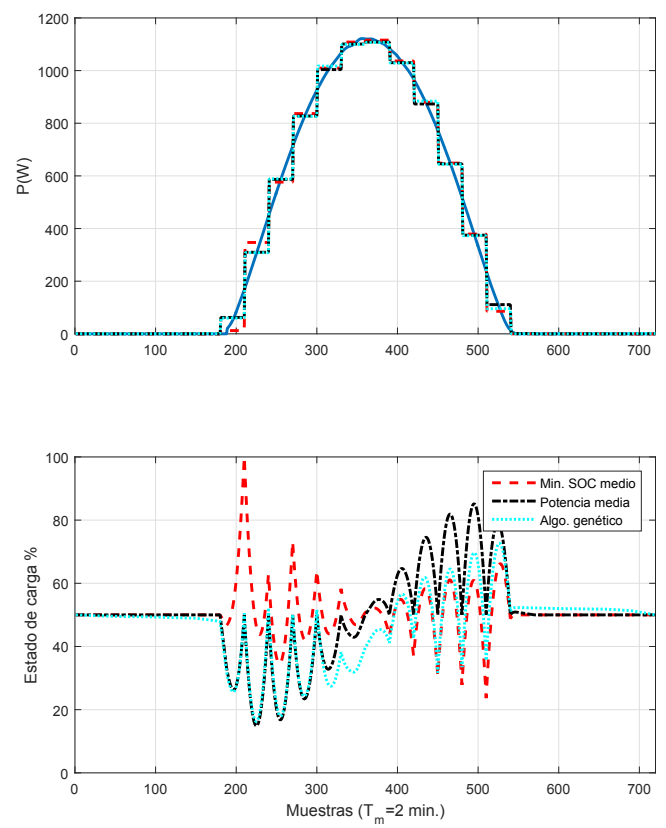

Figura 6: Potencias y estados de carga, mes de marzo

Si se vuelve a calcular el envejecimiento para los 12 días tipos de los 12 meses, se obtienen los resultados que se muestran en la figura 7. Puede comprobarse que el AG mejora en todos los casos a ambos algoritmos.

A continuación, se puede calcular el desgaste de la batería que se produciría a lo largo de todo un año si, para todos los días de cada mes, se tuviera una potencia FV igual que la del día tipo. Aunque se trata obviamente de una suposición que no se cumplirá, sí puede servir para estimar la diferencia del desgaste que se consigue con cada una de las estrategias, Tabla 2.

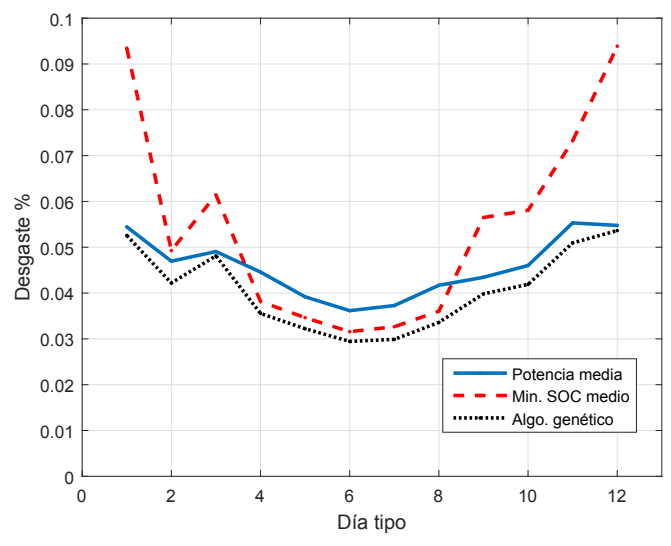

Figura 7: Desgaste de batería para las estrategias básicas y AG

\begin{tabular}{|c|l|c|}
\hline & $\begin{array}{l}\text { Desgaste } \\
\text { anual (\%) }\end{array}$ & Vida(años) \\
\hline P. media & 16.70 & 5.99 \\
\hline Min. SOC & 20.08 & 4.98 \\
\hline Alg. Genético & 15.01 & 6.66 \\
\hline
\end{tabular}

Tabla 2: Desgaste anual en \% y vida estimada

\section{CONCLUSIONES}

En este trabajo se ha propuesto una estrategia óptima para la determinación de una secuencia de 24 escalones de potencia constantes que permitan la participación de una planta FV con batería en el mercado eléctrico. La optimización, formulada mediante algoritmos genéticos, se ha planteado con el objetivo de producir un envejecimiento mínimo de la batería, calculado mediante el algoritmo del Rainflow counting. Ante los resultados de simulación obtenidos a partir de una base de datos de radiación, se puede concluir que gracias al AG se obtiene una reducción de entorno al $10 \%$ en el desgaste de las baterías con respecto a la mejor de las propuestas inicialmente planteadas.

\section{Agradecimientos}

Los autores quieren agradecer a la Universitat Jaume I el apoyo recibido y materializado en el proyecto $\mathrm{P} 1 \cdot 1 \mathrm{~B} 2015-36$.

\section{Referencias}

[1] G. Pepermans, J. Driesen, D. Haeseldonckx, R. Belmans, and W. D'haeseleer, "Distributed generation: definition, benefits and issues," Energy policy, vol. 33, no. 6, pp. 787$798,2005$.

[2] I. PVPS, "International energy agencyphotovoltaic power systems program- 
me,(2008)," Trends in Photovoltaic applications.

[3] R. M. Dell and D. A. J. Rand, "Energy storage-a key technology for global energy sustainability," Journal of Power Sources, vol. 100, no. 1, pp. 2-17, 2001.

[4] S. Jain and V. Agarwal, "An integrated hybrid power supply for distributed generation applications fed by nonconventional energy sources," IEEE Transactions on Energy Conversion, vol. 23, no. 2, pp. 622-631, 2008.

[5] D.-J. Lee and L. Wang, "Small-signal stability analysis of an autonomous hybrid renewable energy power generation/energy storage system part i: time-domain simulations," IEEE Transactions on Energy Conversion, vol. 23, no. 1, pp. 311-320, 2008.

[6] F. Giraud and Z. M. Salameh, "Analysis of the effects of a passing cloud on a gridinteractive photovoltaic system with battery storage using neural networks," IEEE Transactions on Energy Conversion, vol. 14, no. 4, pp. 1572-1577, 1999.

[7] L. Joerissen, J. Garche, C. Fabjan, and G. Tomazic, "Possible use of vanadium redox-flow batteries for energy storage in small grids and stand-alone photovoltaic systems," Journal of Power Sources, vol. 127, no. 1, pp. 98104, 2004.

[8] H. Beltran, E. Bilbao, E. Belenguer, I. Etxeberria-Otadui, and P. Rodriguez, "Evaluation of storage energy requirements for constant production in pv power plants," IEEE Transactions on Industrial Electronics, vol. 60 , no. 3, pp. 1225-1234, 2013.

[9] G. Wang, M. Ciobotaru, and V. G. Agelidis, "Power smoothing of large solar pv plant using hybrid energy storage," IEEE Transactions on Sustainable Energy, vol. 5, no. 3, pp. 834-842, 2014.

[10] H. Gaztañaga, J. Landaluze, I. EtxeberriaOtadui, A. Padrós, I. Berazaluce, and D. Cuesta, "Enhanced experimental pv plant grid-integration with a mw lithium-ion energy storage system," in 2013 IEEE Energy Conversion Congress and Exposition. IEEE, 2013, pp. 1324-1329.

[11] S. Mohammed and A. Muwaffaq, "Restructured electrical power systems, operation, trading, and volatility," 2001.
[12] N. Aparicio, I. MacGill, J. R. Abbad, and H. Beltran, "Comparison of wind energy support policy and electricity market design in europe, the united states, and australia," IEEE transactions on Sustainable Energy, vol. 3, no. 4, pp. 809-818, 2012.

[13] B. Dunn, H. Kamath, and J.-M. Tarascon, "Electrical energy storage for the grid: a battery of choices," Science, vol. 334, no. 6058, pp. 928-935, 2011.

[14] C. Zhou, K. Qian, M. Allan, and W. Zhou, "Modeling of the cost of ev battery wear due to v2g application in power systems," IEEE Transactions on Energy Conversion, vol. 26, no. 4, pp. 1041-1050, 2011.

[15] S. Han, S. Han, and H. Aki, "A practical battery wear model for electric vehicle charging applications," Applied Energy, vol. 113, pp. 1100-1108, 2014.

[16] G. Ning and B. N. Popov, "Cycle life modeling of lithium-ion batteries," Journal of The Electrochemical Society, vol. 151, no. 10, pp. A1584-A1591, 2004.

[17] S. B. Peterson, J. Apt, and J. Whitacre, "Lithium-ion battery cell degradation resulting from realistic vehicle and vehicle-to-grid utilization," Journal of Power Sources, vol. 195, no. 8, pp. 2385-2392, 2010.

[18] G. Ning, R. E. White, and B. N. Popov, "A generalized cycle life model of rechargeable li-ion batteries," Electrochimica acta, vol. 51, no. 10, pp. 2012-2022, 2006.

[19] A. Niesłony, "Determination of fragments of multiaxial service loading strongly influencing the fatigue of machine components," Mechanical Systems and Signal Processing, vol. 23, no. 8, pp. 2712-2721, 2009.

[20] H. Beltran, E. Perez, N. Aparicio, and P. Rodriguez, "Daily solar energy estimation for minimizing energy storage requirements in pv power plants," IEEE Transactions on Sustainable Energy, vol. 4, no. 2, pp. 474-481, 2013.

[21] M. A A $\tilde{A}^{\circ}$ ri, T. A. Huld, and E. D. Dunlop, "Pv-gis: a web-based solar radiation database for the calculation of pv potential in europe," International Journal of Sustainable Energy, vol. 24, no. 2, pp. 55-67, 2005. [Online]. Available: http://dx.doi.org/10.1080/14786450512331329556

$[22]$ C. R. Houck, J. Joines, and M. G. Kay, "A genetic algorithm for function optimization: a matlab implementation," NCSU-IE TR, vol. 95, no. 09, 1995. 